\title{
Self-Optimized Multihop Routing Protocol founded at Wireless Sensor Networks Cross Layer Architecture
}

\author{
Prof. Homera Durani, Prof. Kaushik I. Manavadariya, \\ R.K.University, Tramba \\ Atmiya Institute of Technology \& Science, Rajkot
}

\begin{abstract}
Nowadays, wireless sensor networks (WSNs) are evolving growth wise beneficial, worthwhile and a challenging learning streams. The advancements in WSN endow a broad variety of ecological monitoring and object tracking applications. Moreover, multihop (node by node) routing in WSN is influenced by new instruments certainly entering or departing the network. Thus, nature motivated self-progress and maintained protocols are needed to tackle the problems originating in WSN. We suggested ant colony stimulated routing, which is displaying an extra ordinary performance for WSNs. In this manuscript, a traverse layer conceive founded self-optimized (ACO) routing protocol for WSN and the results are offered. Velocity, power and progress level and Link value parameters are used to find out a minimum route. The power pointer, remaining power and timestamp metrics are trade in from personal level to topology level. The produce and discharge decision through the WSN breakthrough will generate the optimal path from source to destination. The taken up transport or traverse level architecture helps ACO in advancing the overall facts and figures delivery ratio; individually in the case of genuine time traffic.
\end{abstract}

Keywords: Ant Colony Optimization, Cross Layer, Energy, Multihop, Packet Reception Rate, Routing, Velocity, Wireless sensor Network.

\section{Introduction:}

Wireless connection performances a significant role in tele-connection part and has huge significance for the future study. Wireless communications enables many new submissions for sensing and supervising schemes. Some infrastructure free systems like WSN serves an imperative task in monitoring. With the route of time new gadgets and softwareadvancements are evolving available to end-users on a common cornerstone. The stated very quick development andthe gigantic number of apparatus in the mesh make WSN more and more difficult.

The deployment procedure forWSNs is mostly out of the human reach. The overhead cited challenges, such as growing complexity and unreachable upkeep need new answers. The new self-maintained mechanism may refer the characteristics of WSNs such as multihop routing and dynamicallyenvironmental changes in an absolutely autonomous mode. In alignment to address autonomous eligibility for multihopWSNs, it has been shown that self-maintained mesh submissions may leave the operational objectives of the mesh.

In general, probable methods that provide scalability may be found in environment and acclimatized to technology.Towards this vision, it is discerned that various biological principles are adept to overwhelming the overhead adaptabilityproblems. The locality of bio-inspired network technology has the most well renowned advances which are swarm intelligence (ANT Colony, element swarm), AIS and intercellular data exchange[14]. Wireless sensor network routing algorithms based on ANT Colony Optimization (ACO) have been executed in the last couple of years, such as [5], Sensor- driven Cost-aware Ant Routing (SC), the inundated ahead Ant Routing algorithm, and a Flooded Piggybacked Ant Routing (FP) algorithm [6], Adaptive ant-based Dynamic Routing (ADR) [7], Adaptive Routing (AR) and Improved Adaptive Routing (IAR) algorithm [8], and E\&D ANTS [9].

The problems of the preceding in advances is that the selected smallest path might not be a minimum power cost route.Some other focus on declining the power energy utilisation by restoring the hop-count routing with smallest energyrouting. They compute a minimum-energy route for packet delivery in a multi-hop wireless topology protocol. Although, the nodeson this route will get depleted shortly [10]. Radhika D.Joshi [11] granted a concept about combination of least hops andminimum remaining power.

This manuscript present an innovative architecture by applying the most well-known and successful approaches. ACOprocedure is utilized for the optimum path discovery in multihop WSN. Benchmark ACO is very convoluted and heavy forWSN. Consequently, we arrive up with an ACO that can present better optimization for WSN in periods of less burden,less power utilisation and high consignment rate. usually, the 
ACO algorithm is agent founded [12] as ahead ant (FA), backward ant (BA), seek ant (SA) and facts and figures ant (DA) agents as shown in number 1.

\subsection{Outline of Ant Colony Optimization}

\section{Comparative Study}

Dorigo et al suggested the first ant colony algorithms as a multi-agent approach to difficult combinatorial optimization troubles like the traveling salesman problem (TSP) and the quadratic allotment problem (QAP), smallest Weight Vertex Covering difficulty $[15,16]$, and subsequent presented the ACO metaheuristic by Dorigo et al [5].

There are two kinds of ants directed in the algorithms, forward ants and backward ants. Forward ants, whose major actions are discovering the route and collecting the data from the source nodes to destination visited node, it have the numeric identity as the source nodes. The paths that ahead ants journey will assemble a tree when they amalgamate into each other or come to the place visited and data is conveyed along the tree routes.

There are two key components that perform the action of the ahead ants: one is pheromone trails that are made a down payment along the perimeters, and the other is the nodes promise which provides an estimate of how far an ant will have to travel from any the node to either come to the place visited or to aggregate data with another node. While the in reverse ants, traveling back from place visited node to source nodes opposing to the ahead ants, present their uppermost function of updating the data of their pass-by nodes.

ACO algorithms are a class of constructive meta-heuristic algorithms that imitate the cooperative demeanour of real ants to accomplish convoluted computations and have been verified to be very efficient to numerous distinct discrete optimizationproblems. Many theoretical analyses associated to ACO display that this optimization can converge to the internationaloptima with non-zero positive likelihood in the answer space and their presentation have greatly matched numerous well-studied stochastic optimization algorithms, for demonstration, genetic algorithm, pattern search, GPASP, and annealing simulations .

\subsection{Evaluation between Ant based Routing means}

Evaluation of the latest ANT based routing in WSN: SC and [19] depends on the power metric while FF founded on delay. IA and IAR is the modification of ADR which utilised a hold up parameter in the lines to approximate reinforcement discovering factor. In FP they blend the ahead ant and facts and figures ant to enhance the achievement rate. E\&D ANT founded on energy and delay metrics for routing procedures.

In our proposed algorithm, the best standards of velocity, PRR and residual power mechanism [20] are utilised to choose forwarding node because velocity alone does not provide the data about link value. The best link value generally provides low package decrease and power effective [21]. Another novel characteristic is the remaining power parameter to select the forwarding candidate node.

\subsection{Comparison between Traverse levels founded Mechanisms}

During the preceding ten years, numerous power efficient network routing protocols have been suggested for sensor systems. Earlier routing schemes engage direct connection protocols which facilitate direct connection between the source node and the groundwork position. Thus, for scenarios where the groundwork position is quite an expanse away from the source node, there is excessive usage of power assets, finally producing in a complete drainage of power. Such routing designs are successful only where sensor nodes are beside enough to the groundwork position [22].

In $[10,23]$, an Adaptive NAV-Assisted Routing (ANAR) protocol is proposed to alleviate the mesh congestion founded onthe cross-layer data. The ANAR design utilizes the existing data from the Request-ToSend (RTS) and the Clear-to-Send(CTS) packets within the contention founded MAC design.

In [24], founded on the received signal power, they can outlook if the mobile node will move out of the transmission scope shortly, and change to the next node before the connection broken. It collects the MAC level residual bandwidth,connection hold up and battery remaining power information, then utilizes cross-layer communication to changepheromone worth counting on the heaviness worth of QoS parameters, to reduce package decrease and choose an optimal route.Suggested a traverse-layer architecture utilising MAC and Routing level.

The traverse layer architecture applied inthis study is characterised by the interaction of 802.11 MAC protocol and the Dynamic Source Routing protocol. Thecross-layer architecture applied in this work will be able to reduce routing overheads, by decreasing the route administrationprocessing presented by the DSR protocol in most scenarios. The authors have applied the 802.11 MAC additions in mesh Simulator-2 (NS-2) that stores the last pointer strength obtained from all neighbouring nodes. In supplement, they furthermore modified the 802.11 MAC layer to drive a message to the top level in case there is a decrease of connection but the place visited node is still within the transmission variety. 


\subsection{Overview of ACO based Routing Algorithm in WSN}

Researcher has granted an on-line ACO algorithm using AntNet techniques for MSDC [18] which has been formalized to be a typically smallest Steiner Tree problems. They furthermore have proposed an improved algorithm by supplementing another kind of ants, random ants, analogous to a bulletin deliverer, whose major task is to disperse data accumulated at the nodes among other neighbouring nodes. Virtually, replication outcomes also display that their algorithms are significantly better than address-centric routing. In these suggested algorithms the ahead ants commonly spend a long time. There is a bug of dead secure in their algorithms. In their improved algorithm, a large number of random ants are required.

In [5] the authors suggest a new concept of holding the information by all sensor nodes of their own. By this even in the nonattendance of international processing the nodes still can work on their own data. This still has the drawback of broadcasting in the initialization stage, which consumes allotments of energy at the starting of the mesh deployment. Zhang et al. [6] proposed three ant-routing algorithms for sensor systems. The $\mathrm{SC}$ algorithm is power effective but suffers from a reduced success rate. The FF algorithm has shorter time delays; although, the algorithm conceives a important allowance of traffic. Despite high success rate shown by the FP algorithm but is not energy effective.

An Adaptive ant-based Dynamic Routing (ADR) algorithm using a novel variation of reinforcement discovering was proposed by Lu et al. [7]. The authors used a delay parameter in the queues to approximate the reinforcement learning component. Karaboga [19] suggested a novel approach for WSN routing procedures. Through this approach the network life time is maximized, for finding out the shortest paths from the source nodes to the base node utilising an evolutionary optimization method. The study has also been implemented on the PIC series of micro controllers.

Aghaei et al [8] proposes two adaptive routing algorithms founded on ant colony algorithm, the Adaptive Routing (AR) algorithm and the Improved Adaptive Routing (IAR) algorithm. To ascertain the suitability of the ADR algorithm in the case of sensor networks, they changed the ADR algorithm (removing the line parameters) and utilised their reinforcement discovering notion and named it the AR algorithm. The AR algorithm did not outcome in minimum answers. In IAR algorithm by supplementing a coefficient, the cost between the neighbor node and the place destination node, they further advance the AR algorithm.

Researcher [9] are suggested a dynamic adaptive ant algorithm (E\&D ANTS) is based on power and Delay metrics for routing procedures. Their main goal is to maximize the mesh lifetime while minimizing propagation hold up by utilising a novel variety of reinforcement learning (RL). E\&D ANT's outcomes was evaluated with AntNet and AntChain schemes.

\section{Cross-Layer conceive}

The notion of cross-layer conceive is about sharing of data amidst two or more levels for adaptation purposes and to increase the inter-layer interactions [24, 29, and 30]. The proposed scheme utilises interaction between the physical level and the mesh level in order to choose the best next node as shown in Figure 2.

In this manuscript, we have suggested an enhanced ant colony motivated self-optimized routing protocol for WSN. Our specified means is founded on connection value, energy and velocity parameters. The adopted traverse level architecture assists WSN in improving the general data throughput; particularly in the case of real time traffic. The traverse layer conceive furthermore assists WSN in better consignment ratio while sustaining power utilisation. The algorithm is also adept of bypassing enduring loops which encourages dead lock in the running systems. Replication and untested results illustrate the protocol efficiency. Finally, this autonomic routing means will arrive up with better delivery ratio over WSN. Our direct future work developed to enhance our routing means with autonomous security system. The method at the mesh layer comes up with the optimal conclusion founded on the personal parameters, which are then converted as forwarding metrics. The came by physical parameters are pointer power, remaining power and timestamp. The forwarding metrics are utilised to get an optimal conclusion. The forwarding mechanism is demanded only during close by discovery and network initialization phase.

\section{Conclusion}

In this manuscript, we have proposed an enhanced ant colony inspired self-optimized routing protocol for WSN. Our particular means is founded on connection value, power and velocity parameters. The adopted traverse layer architecture assists WSN in improving the overall data throughput; particularly in the case of genuine time traffic. The cross layer conceive furthermore assists WSN in better delivery ratio while maintaining power consumption. The algorithm is furthermore capable of bypassing permanent loops which encourages dead secure in the running systems. Replication and untested results demonstrate the protocol effectiveness. Finally, this autonomic routing means will arrive up with better delivery ratio over WSN. Our direct future work developed to enhance our routing mechanism with autonomous security system. 


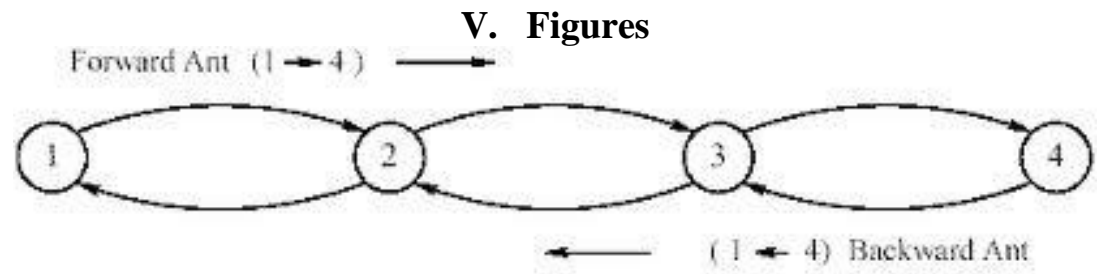

Fig 1 - Forward and Backward Ant

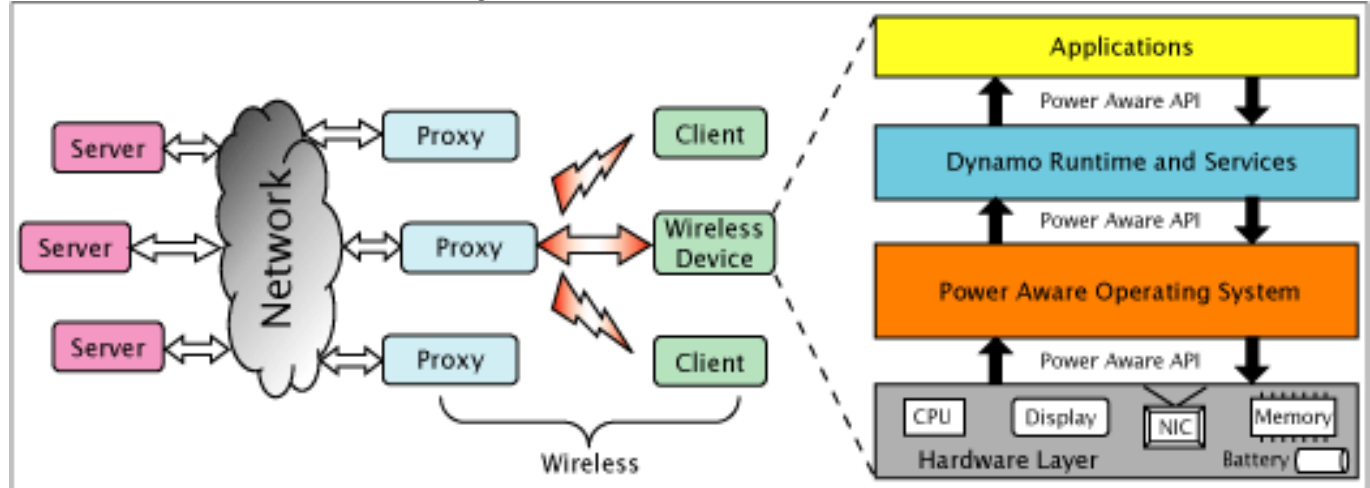

Fig 2 - Architecture Cross Layer

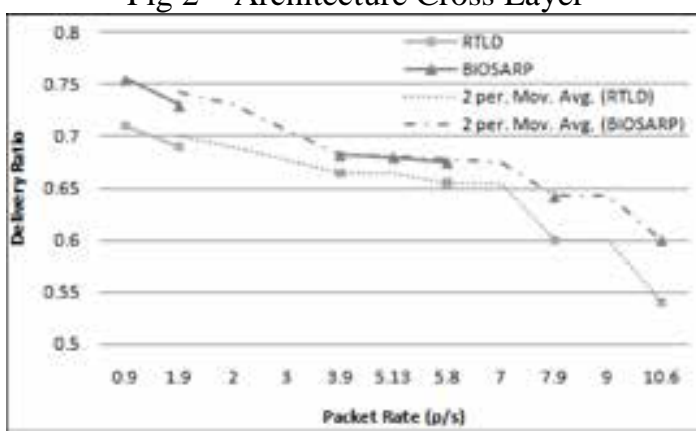

Fig 3 - Delivery of Distinct Package Rate

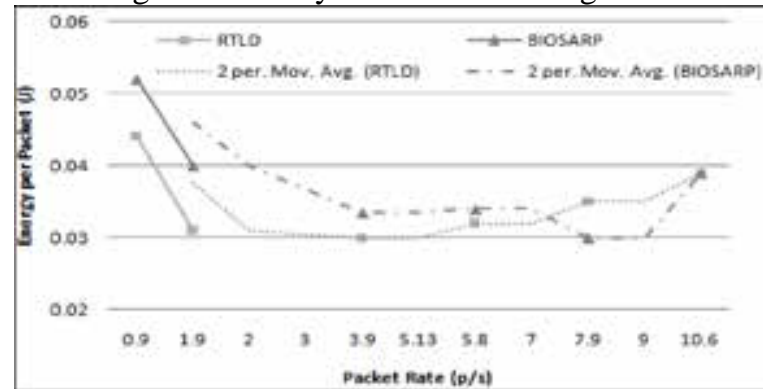

Fig 4 - Power per Package

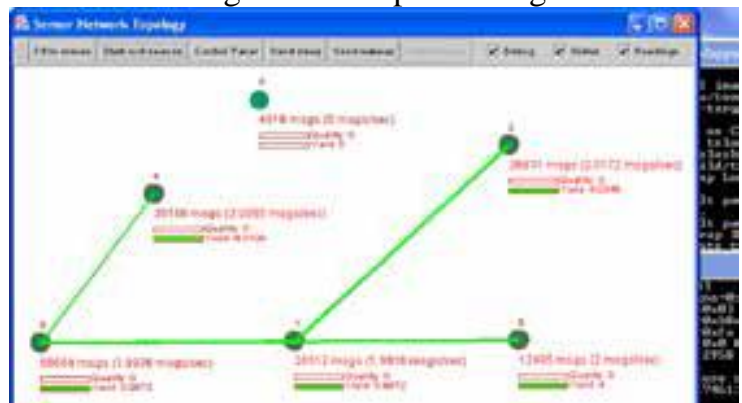

Fig 5 - Network tested 


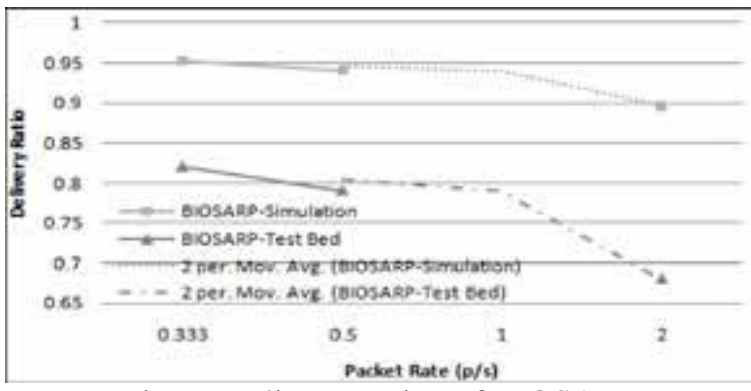

Fig 6 - Delivery Ration of BIOSARP

\section{References}

[1] S. Balasubramaniam, D. Botvich, W. Donnelly, M. Foghluh, and J. Strassner, "Biologically Inspired Self-Governance and SelfOrganisation for Autonomic Networks," in Proceedings of the 1st international conference on Bio inspired models of network, information and computing systems. vol. 275Cavalese, Italy: ACM, 2006, p. 30.

[2] S. Balasubramaniam, W. Donnelly, D. Botvich, N. Agoulmine, and J. Strassner, "Towards integrating principles of Molecular Biology for Autonomic Network Management," in Hewlett Packard university Association (HPOVUA) conference, Nice, France., 2006.

[3] P. Boonma and J. Suzuki, "MONSOON: A CoevolutionaryMultiobjective Adaptation Framework for Dynamic Wireless Sensor Networks," in In Proc. of the 41st Hawaii International Conference on System Sciences (HICSS) Big Island, HI, 2008.

[4] N. Mazhar and M. Farooq, "BeeAIS: Artificial Immune System Security for Nature Inspired, MANET Routing Protocol, BeeAdHoc," Springer-Verlag Berlin Heidelberg, vol. LNCS 4628, pp. 370-381, 2007.

[5] G. Chen, T.-D. Guo, W.-G. Yang, and T. Zhao, "An improved ant-based routing protocol in Wireless Sensor Networks," in Collaborative Computing: International Conference on Networking, Applications and Worksharing, 2006. CollaborateCom2006., New York, NY, 2006, pp. 1-7.

[6] Y. Zhang, L. D. Kuhn, and M. P. J. Fromherz, "Improvements on Ant Routing for Sensor Networks," M. Dorigo et al. (Eds.): ANTS 2004, Springer-Verlag Berlin Heidelberg 2004, vol. LNCS 3172, pp. 154-165, 2004.

[7] Y. Lu, G. Zhao, and F. Su, "Adaptive Ant-based Dynamic Routing Algorithm," in In Proceedings of the 5th World Congress on Intelligent Control and Automation, Hangzhuo, China, 2004, pp. 2694-2697.

[8] R. G. Aghaei, M. A. Rahman, W. Gueaieb, and A. E. Saddik, "Ant Colony-Based Reinforcement Learning Algorithm for Routing in Wireless Sensor Networks," in Instrumentation and Measurement Technology Conference - IMTC Warsaw, Poland: IEEE, 2007.

[9] Kaushik I. Manavadariya, Deven J. Patel and Kapil K. Shukla, “COMPARATIVE STUDY OF Bluetooth, 802.11 and Hiperlan" in IAEME (IJCET) Issue 3, Volume 4, 2013.

[10] W. Wang, D. Peng, I.-H. Youn, H. Wang, and H. Sharif, "Cross Layer Design and Implementation for Balancing Energy Efficiency in Wireless Sensor Networks," Information Technology Jornal, pp. 648-655, 2007.

[11] R. D.JOSHI and P. P.REGE, "Energy Aware Routing in Ad Hoc Networks," in 6th WSEAS International Conference on CIRCUITS, SYSTEMS, ELECTRONICS, CONTROL \& SIGNAL PROCESSING, Cairo, Egypt, 2007, pp. 469-475.

[12] M. S. Kakkasageri, S. S. Manvi, and G. D. Soragavi, "Mobile agent based event discovery in wireless sensor networks," in Proceedings of the 5th WSEAS International Conference on Applied Computer Science, Hangzhou, China, 2006, pp. 731-735.

[13] G. D. Caro, "Ant Colony Optimization and its Application to Adaptive Routing in Telecommunication Networks," In FACULTE DES SCIENCES APPLIQUEES. vol. Docteur en Sciences Appliquees | BRUXELLES: UNIVERSITE LIBRE DE BRUXELLES, 2004, p. 374.

[14] S. L. Y. B. M. S. Q. D. D. Qian, "CLEEP: A Novel Cross-Layer Energy-Efficient Protocol for Wireless Sensor Networks," in 4th International Conference on Wireless Communications, Networking and Mobile Computing, 2008. WiCOM '08, 2008, pp. 1-4.

[15] T. Milan and J. Raka, "An analysis of different variations of ant colony optimization to the minimum weight vertex cover problem," WSEAS Trans. Info. Sci. and App., vol. 6, pp. 936-945, 2009.

[16] R. Jovanovic, M. Tuba, and D. Simian, "Comparison of Different Topologies for Island-Based Multi-Colony Ant Algorithms for the Minimum Weight Vertex Cover Problem," WSEAS TRANSACTIONS on COMPUTERS, vol. 9, January 2010.

[17] T. Stuetzle and M. Dorigo, "A Short Convergence Proof for a Class of ACO Algorithms," IEEE Transactions on Evolutionary Computation, vol. 6, pp. 358-365, 2002.

[18] G. Singh, S. Das, S. Pujar, and S. Gosavi, "Ant Colony Algorithms for Steiner Trees: An application to Routing in Sensor Networks," in Recent Developments in Biologically Inspired Computing, F. J. v. Z. L. N. de Castro, Ed.: Idea Group Publishing, 2004, pp. 181-206.

[19] S. Okdem and D. Karaboga, "Routing in Wireless Sensor Networks Using Ant Colony Optimization," in Proceedings of the First NASA/ESA Conference on Adaptive Hardware and Systems (AHS'06), Istanbul, 2006.

[20] A. Ali, L. A. Latiff, M. A. Sarijari, and N. Fisal, "Real-time Routing in Wireless Sensor Networks," in The 28th International Conference on Distributed Computing Systems Workshops, Beijing, China, 2008. 